\title{
Silicon Photomultipliers: Properties, Latest Developments at FBK and Applications.
}

\section{Claudio Piemonte ${ }^{1}$}

${ }^{1 .}$ Fondazione Bruno Kessler (FBK), Center for Materials and Microsystems, Trento, Italy.

The Silicon photomultiplier ( $\mathrm{SiPM})$ is a novel technology showing a rapidly growing interest in many areas requiring the detection of faint light. The development of these devices started in the end of the nineties at MEPHI (Moscow, Russia) and has seen significant technological progress, in particularly in the last decade. The SiPM is manufactured using CMOS-compatible technologies. It has a typical size in the millimeter scale and comprises a densely packed matrix of small (from 10 to 100 microns size) Single-Photon Avalanche Photodiodes (SPADs) with individual quenching resistors. These SPADs are connected in parallel so that the single output signal is proportional to the number of elements hit by photons (Figure 1a). The charge packet delivered by a single SPAD when fired by a photon is in the order of one million electrons, which is very similar to Photomultiplier Tubes (PMTs). Some important advantages offered by this solid-state solution with respect to the classic PMTs are: compactness, low operating voltage, ruggedness, insensitivity to magnetic fields and long lifespan. With respect to their application in electron imaging, fast recovery, large dynamic range, and the ability to produce them in custom geometries, are important characteristics. Finally, the current high level of competition in the market is pushing the pace of development and cost reduction.

In operation conditions, the SPADs are biased through the quenching resistor at a voltage higher than the breakdown ( $\mathrm{V}_{\mathrm{BD}}$ ) and (ideally) no current is flowing. $\mathrm{V}_{\mathrm{BD}}$ is in the range of 25 to $50 \mathrm{~V}$, whereas the bias voltage is $5-10 \mathrm{~V}$ above this value (over-voltage). When a photon hits a SPAD, the junction discharges to $\mathrm{V}_{\mathrm{BD}}$ delivering an amount of charge proportional to the capacitance and the over-voltage. Then, the SPAD recovers its initial bias condition through the quenching resistor. The typical singlephoton signal shape features a very fast rising edge (few hundreds of ps) allowing for very precise timing and an exponential trailing edge with a time constant of few tens of ns.

In dark conditions, thermally generated carriers may trigger a discharge not distinguishable from a photon-triggered one. The rate of these events, called dark count rate (DCR), is the device noise. Latest products feature a DCR between 10 and $100 \mathrm{kHz} / \mathrm{mm}^{2}$ at room temperature. Two other noise components affect the device and they are correlated to the presence of a primary signal, either a dark or a light event. The first is the optical cross-talk: during a SPAD discharge, photons are emitted and they might trigger other unwanted avalanches in neighbouring SPADs. Latest technologies feature trenches filled with absorbing or reflecting material to attenuate this phenomenon. The second is after-pulsing. This noise event is a discharge triggered by a carrier which was trapped during a previous avalanche and then released with a certain delay. After-pulsing has been reduced to few percent in latest devices mainly selecting proper silicon material.

The last important parameter is the Photon-Detection Efficiency (PDE). It is the product of three components: the SPAD active-to-total area ratio (fill factor, FF), the quantum efficiency (QE), and the avalanche triggering probability $(\mathrm{Pt})$. The latter factor is the probability for a carrier travelling in the high-filed region of the SPAD to trigger an avalanche. The best performing SiPMs have the efficiency peaked in the blue/nearUV. Also the PDE has improved dramatically in the past few years: FF and QE are close to $80 \%$ while Pt approaches $100 \%$, giving an overall efficiency exceeding 50\%. Figure 2 (left) 
shows an example of the PDE shape of the latest NUV-HD samples (developed at FBK) having a SPAD pitch of $30 \mu \mathrm{m}$ [1]. SiPMs can have peak efficiency in the green/yellow region as well, such as the RGBHD produced by FBK.

One of the most common applications of SiPMs is the detection of the light emitted by scintillators. They are widely used in Time-of-Flight Positron Emission Tomography (TOF-PET) providing much better performance compared to PMTs especially in terms of timing resolution, position resolution and integration [1].

Besides further improving the basic performance, the SiPM technology is evolving in different directions to meet other requirements. One example is the extension of the efficiency spectrum in the extreme UV, e.g. for the detection of scintillation light from liquid Xe. This technology can open the way to efficient direct electron detection as well. Another development carried out at FBK is a ultrahigh-density-SPAD SiPM with many purposes: to increase the dynamic range, to have a shorter signal and better radiation tolerance. Latest devices produced have a SPAD pitch down to $7.5 \mu \mathrm{m}$. In Figure 2, an SEM picture of a ultra-high-density device is shown along with a single photon spectrum.

The author acknowledges the colleagues in [2].

References:

[1] C. Piemonte et al, IEEE Transactions on Electron Devices DOI: 10.1109/TED.2016.2516641.

[2] F. Acerbi, A. Ferri, A. Gola, G. Paternoster, V. Regazzoni, G. Zappala', N. Zorzi are thanked for the substantial contribution on the SiPMs activities at FBK.
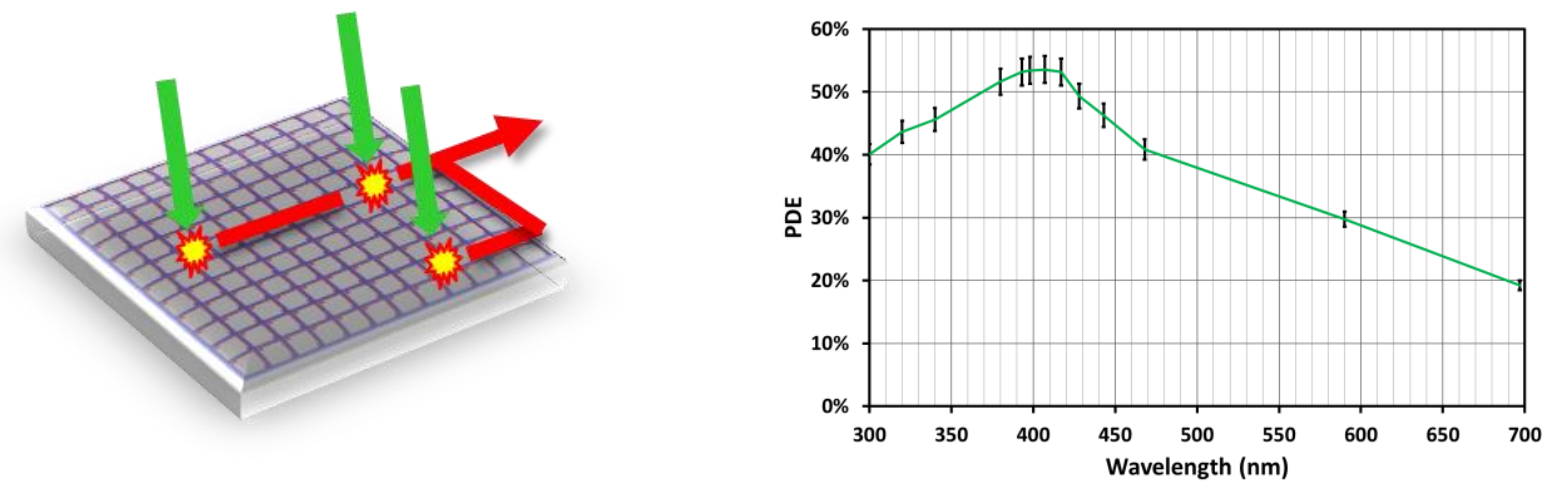

Figure 1. Left: sketch of the working principle of a SiPM. Right: photon-detection efficiency measured on the latest prototypes of NUV-HD SiPMs with $30 \mu \mathrm{m}$ SPAD pitch.
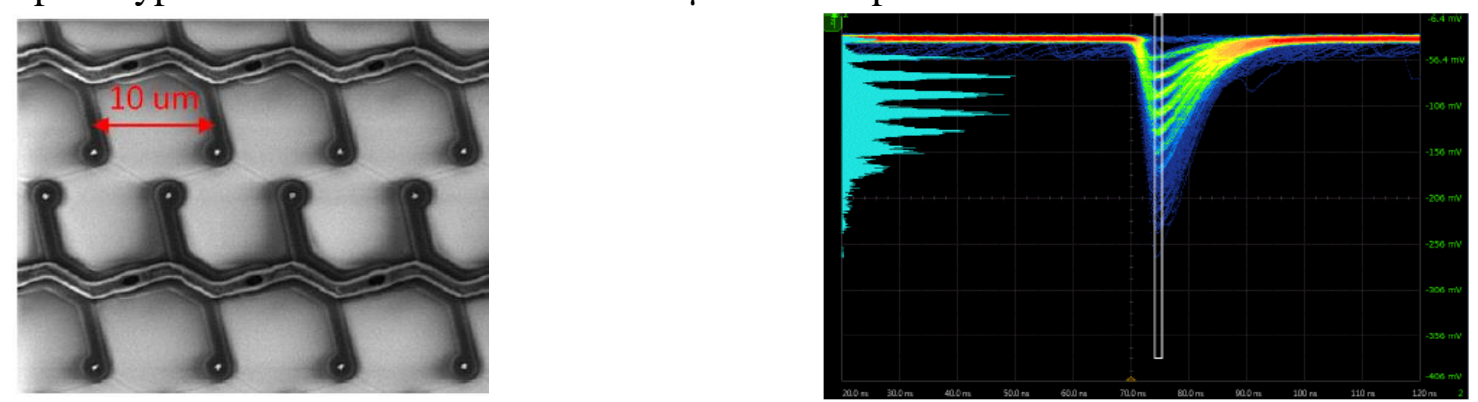

Figure 2. Left: SEM picture of a SiPM with $10 \mu \mathrm{m}$ pitch SPADs. Right: single-photon spectrum of the SiPM with $10 \mu \mathrm{m}$ pitch SPAD. 